\title{
MIMO Broadcast Channels with Digital Channel Feedback
}

\author{
Nihar Jindal \\ Department of Electrical and Computer Engineering \\ University of Minnesota \\ Minneapolis, MN 55455, USA
}

\begin{abstract}
This paper summarizes recent results on the multiple antenna broadcast (downlink) channel in which limitedrate digital feedback is used to convey channel state information from each mobile (receiver), each of which is assumed to have perfect channel information, to the transmitter. The transmitter employs linear precoding based on this feedback, and the effect of imperfect channel state information is multi-user interference, which can significantly degrade throughput if the channel information is not sufficiently accurate. Feedback requirements are studied for systems with single antenna mobiles in both the small system regime, i.e., number of mobiles equal to the number of transmit antennas, as well as in the large system regime, where user selection is performed on the basis of digital feedback. In addition, two different methods for utilizing multiple mobile antennas are described and compared in the small system regime.
\end{abstract}

\section{INTRODUCTION}

In multiple antenna broadcast (downlink) channels, capacity can be tremendously increased by adding antennas at only the access point (transmitter) if accurate channel state information is available at the transmitter and at receivers [1]. In frequency-division duplexed systems, training can be used to obtain channel knowledge at each of the mobile devices (receivers), but obtaining CSI at the access point generally requires feedback from each mobile. In the practically motivated limited-rate digital feedback model, each mobile feeds back a finite number of bits regarding its channel instantiation at the beginning of each block. This model was first considered for point-to-point MIMO channels in [2][3][4]. Although a relatively small number of feedback bits is generally sufficient to achieve performance close to perfect CSIT for point-topoint MIMO channels, MIMO downlink channels require considerably higher levels of feedback because imperfect CSIT leads to multi-user interference, which significantly degrades performance.

We consider downlink systems in which simple linear precoding techniques (e.g., zero-forcing beamforming or block diagonalization) are performed on the basis of error- and delay-free digital channel feedback from each mobile. We first consider systems in which each mobile has a single antenna. In the small system regime, i.e., number of mobiles equal to number of transmit antennas, per mobile feedback must scale linearly with the number of transmit antennas and the system SNR in order to achieve throughput close to that of a perfect CSIT system [5]. In systems with a large number of users, user selection algorithms are used to select a subset of users for transmission. We describe the channel information required such that these algorithms can perform effectively and exploit multi-user diversity in the presence of imperfect CSIT [6]. In contrast to analysis of random beamforming technique for an asymptotically large number of users [7], this work indicates that even very large but finite systems (e.g., 4 transmit antennas and 100-1000 mobiles) are quite sensitive to imperfect CSIT, and thus require high-rate channel feedback.

We also consider systems in which each mobile device has multiple antennas, and describe two different methods for utilization of these antennas. Block diagonalization (BD), which is an extension of zero-forcing beamforming that allows multiple data streams to be sent to each mobile [8], [9], can be performed on the basis of appropriate digital channel feedback, and is seen to require less feedback than a naive zero-forcing strategy [10]. A novel antenna combining method, in which combining is performed to reduce quantization error rather than increase received signal power, is seen to lead to feedback requirements that are very similar to those for BD [11].

\section{System MODEL}

We consider a $K$ receiver multiple antenna broadcast channel in which the transmitter (access point or AP) has $M$ antennas and each of the mobiles has $N$ receive antennas. The received signal at the $i$-th mobile is given by:

$$
\mathbf{y}_{i}=\mathbf{H}_{i}^{H} \mathbf{x}+\mathbf{n}_{i}, \quad i=1, \ldots, K
$$

where $\mathbf{H}_{1}, \mathbf{H}_{2}, \ldots, \mathbf{H}_{K}$ are the $M \times N$ channel matrices, the vector $\mathbf{x} \in \mathbb{C}^{M \times 1}$ is the transmitted signal, and $\mathbf{n}_{1}, \ldots, \mathbf{n}_{K}$ are independent complex Gaussian noise vectors with iid unit variance components. There is a transmit power constraint of $P$, i.e., the input must satisfy $E\left[\|\mathbf{x}\|^{2}\right] \leq P$.

We consider a block fading channel, with independent Rayleigh fading from block to block (i.e., the components of the channel vectors are iid unit variance complex Gaussian). Each of the receivers is assumed to have perfect and instantaneous knowledge of its own channel matrix $\mathbf{H}_{i}$. It is not necessary for mobiles to know the channel of other mobiles.

\section{A. Digital Channel Feedback Model}

At the beginning of each block, each receiver quantizes its channel to $B$ bits and feeds back the bits perfectly and instantaneously to the access point. When each mobile has a single antenna $(N=1)$, vector quantization is performed 
using a codebook $\mathcal{C}$ that consists of $2^{B} M$-dimensional unit norm vectors $\mathcal{C} \triangleq\left\{\mathbf{w}_{1}, \ldots, \mathbf{w}_{2^{B}}\right\}$. Each receiver quantizes its channel vector to the quantization vector that forms the minimum angle to it [3] [4]. Thus, user $i$ quantizes its channel to $\hat{\mathbf{h}}_{i}$, chosen according to:

$$
\hat{\mathbf{h}}_{i}=\arg \min _{\mathbf{w}=\mathbf{w}_{1}, \ldots, \mathbf{w}_{2 B}} \sin ^{2}\left(\angle\left(\mathbf{h}_{i}, \mathbf{w}\right)\right) .
$$

and feeds the quantization index back to the transmitter. It is important to notice that only the direction of the channel vector is quantized.

In this work we use random vector quantization (RVQ), in which each of the $2^{B}$ quantization vectors is independently chosen from the isotropic distribution on the $M$-dimensional unit sphere [12]. Each receiver is assumed to use a different and independently generated codebook, and we analyze performance averaged over the distribution of random codebooks.

When $N>1$, the quantization codebook consists of matrices and the distance metric can be appropriately defined. Furthermore, random quantization corresponds to choosing the quantization matrices independently from the set of all unitary matrices. See Section IV-A for more details.

\section{B. Linear Precoding}

After receiving the quantization indices from each of the mobiles, the AP uses linear precoding to transmit data to the mobiles. When $N=1$, we consider the simple strategy of zero-forcing beamforming (ZFBF). Since the transmitter does not have perfect CSI, ZFBF is performed based on the quantizations instead of the channel realizations. When ZFBF is used, the transmit vector is defined as $\mathbf{x}=\sum_{i=1}^{M} \mathbf{v}_{i} s_{i}$, where each $s_{i}$ is a scalar (chosen complex Gaussian with power $P(M)$ intended for the $i$-th receiver, and $\mathbf{v}_{i} \in \mathcal{C}^{M}$ is the beamforming vector for the $i$-th receiver. The beamforming vectors $\mathbf{v}_{1}, \ldots, \mathbf{v}_{M}$ are chosen as the normalized rows of the matrix $\left[\hat{\mathbf{h}}_{i} \cdots \hat{\mathbf{h}}_{M}\right]^{-1}$, and thus they satisfy $\left\|\mathbf{v}_{i}\right\|=1$ for all $i$ and $\hat{\mathbf{h}}_{i}^{H} \mathbf{v}_{j}=0$ for all $j \neq i$. The resulting SINR at the $i$-th mobile is:

$$
\operatorname{SINR}_{i}=\frac{\frac{P}{M}\left|\mathbf{h}_{i}^{H} \mathbf{v}_{i}\right|^{2}}{1+\sum_{j \neq i} \frac{P}{M}\left|\mathbf{h}_{i}^{H} \mathbf{v}_{j}\right|^{2}} .
$$

The achievable long-term average rate is the expectation of $\log \left(1+S I N R_{i}\right)$ over the distribution of the fading and RVQ.

When $N>1$, ZFBF can be generalized to block diagonalization, as described in Section IV-A.

\section{Single Antenna Mobiles}

In this section we summarize results on MIMO downlink channels in which each receiver has a single receive antenna.

\section{A. Small System Analysis: $K=M$}

We first consider a system in which the number of receivers is equal to the number of transmit antennas. This analysis applies to systems in which the number of users is relatively small, as well as to large systems in which $M$ users are selected for transmission on the basis of quantities independent of their channel feedback, e.g., deadline constraints. In order to characterize the sensitivity of the system to channel feedback, we compare the long-term average throughput with perfect CSIT to the throughput of a feedback-based system.

If the transmitter has perfect CSIT, the beamforming vectors (denoted $\mathbf{v}_{Z F, i}$ ) can be chosen perfectly orthogonal to all other channels, thereby creating a parallel and non-interfering channel to each mobile. Thus the average rate (per mobile) is:

$$
R_{Z F}(P)=E_{\mathbf{H}}\left[\log \left(1+\frac{P}{M}\left|\mathbf{h}_{i}^{H} \mathbf{v}_{Z F, i}\right|^{2}\right)\right] .
$$

With limited feedback, multi-user interference cannot be completely eliminated and the resulting average rate is:

$$
R_{F B}(P)=E_{\mathbf{H}, W}\left[\log \left(1+\frac{\frac{P}{M}\left|\mathbf{h}_{i}^{H} \mathbf{v}_{i}\right|^{2}}{1+\sum_{j \neq i} \frac{P}{M}\left|\mathbf{h}_{i}^{H} \mathbf{v}_{j}\right|^{2}}\right)\right] .
$$

Since each beamforming vector $\mathbf{v}_{j}$ is chosen orthogonal to the quantization vectors $\left\{\hat{\mathbf{h}}_{i}\right\}_{i \neq j}$, a smaller angle (i.e., a finer quantization) between the channel $\mathbf{h}_{j}$ and its quantization $\hat{\mathbf{h}}_{j}$ will lead to smaller interference terms $\left|\mathbf{h}_{i}^{H} \mathbf{v}_{j}\right|^{2}$, and thus a higher rate.

In order to quantify the effect of digital feedback, we define the rate gap $\Delta R(P)$ as:

$$
\Delta R(P) \triangleq R_{Z F}(P)-R_{F B}(P) .
$$

By utilizing the statistics of RVQ and of Rayleigh fading channels, we get the following upper bound on $\Delta R(P)$ :

Theorem 1 ([5]): RVQ-based digital feedback with $B$ bits per mobile incurs a rate gap upper bounded by:

$$
\Delta R(P)<\log _{2}\left(1+P \cdot 2^{-\frac{B}{M-1}}\right) .
$$

The most important feature to notice is that the rate gap is an increasing function of the SNR as well as of $M$. In fact, it can be shown that if $B$ is fixed and the SNR $P$ is taken to infinity, such a system becomes interference-limited [5]. In order to prevent this and to additionally achieve the full multiplexing gain (i.e., a rate vs. SNR curve with the same slope as perfect CSIT), the feedback quality must be appropriately scaled with the SNR. The following theorem, which is derived by simply inverting the rate gap upper bound, quantifies the scaling of feedback needed to keep the rate gap $\Delta(P)$ bounded at all SNR's. This condition also ensures that the full multiplexing gain is achieved.

Theorem 2 ([5]): A rate gap $\Delta(P)$ no larger than a constant $r>0$ is maintained at all SNR's by scaling $B$ as:

$$
\begin{aligned}
B & =(M-1) \log _{2} P-(M-1) \log _{2}\left(2^{r}-1\right) \\
& \approx \frac{M-1}{3} P_{d B}-(M-1) \log _{2}\left(2^{r}-1\right) .
\end{aligned}
$$

Similar to point-to-point MIMO systems, it is necessary to scale feedback approximately linearly with the number of transmit antennas $M$. Unlike point-to-point systems, feedback must also be scaled linearly with the system SNR (in $d B$ ).

In Fig. 1, achievable rates vs. SNR are shown for a 6 antenna, 6 user system, for perfect CSIT zero-forcing, fixed $B=15$ (and thus interference-limited), and with $B$ scaled 


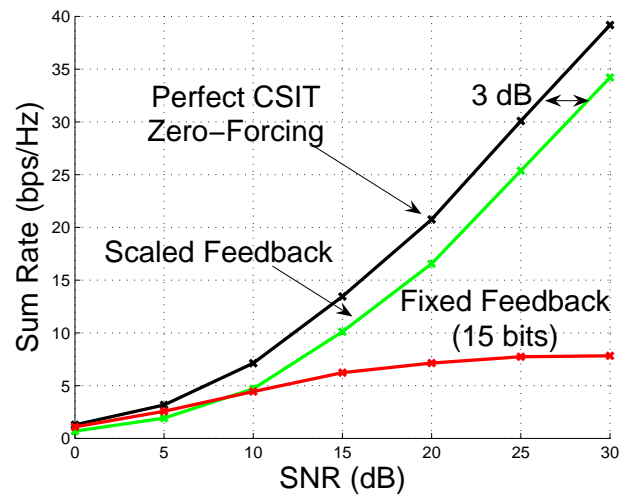

Fig. 1. Downlink Channel with $M=K=6, N=1$

according to (2) with $r=1$, i.e., $B=\frac{M-1}{3} P_{d B}$. Note that $r=1$ corresponds to a rate gap of $1 \mathrm{bps} / \mathrm{Hz}$, which is equivalent to a $3 \mathrm{~dB}$ power shift.

Note that the analysis in this section has been based on the assumption of transmission to all $M$ users and equal power loading. At low and moderate SNR's (e.g., up to 10 $\mathrm{dB}$ ), throughput can be increased non-negligibly by selecting a smaller subset of users for transmission using an algorithm such as in [13], and also by using power allocation if some channel magnitude information is available [14]. However, whenever two or more users are selected for transmission, the feedback requirements are the same (in the scaling sense) as the scenario analyzed here.

\section{B. Large System Analysis: $K>M$}

In systems with many users, throughput can be significantly increased by transmitting to only a selected subset of up to $M$ users. A considerable amount of research has focused on the design of such selection algorithms, which attempt to select users with large channel magnitudes as well as with nearly orthogonal channels, under the assumption of perfect CSIT (e.g., [15][13]). If such an algorithm is used, multiuser diversity can be exploited and throughput grows doublelogarithmically with $K$, even when the SNR is fixed.

In [6], a system performing user selection on the basis of limited channel feedback is studied. In order to select a strong subset of users, the transmitter must be provided with some channel quality information (CQI) in addition to the channel directional information (CDI) contained in the $B$-bit quantization index. The most straightforward approach is to define the CQI to be the norm of each channel, i.e., $\left\|\mathbf{h}_{i}\right\|^{2}$. If the transmitter is provided with perfect knowledge of this CQI (in addition to $B$-bit $\mathrm{CDI}$ ), then somewhat surprisingly the system throughput is bounded as $K \rightarrow \infty$ with $B$ and $P$ fixed. If $K$ is very large, the transmitter is able to find a set of users with very large channel norms and whose quantization vectors are nearly orthogonal. However the quantization error, which the transmitter is unaware of, leads to residual multi-

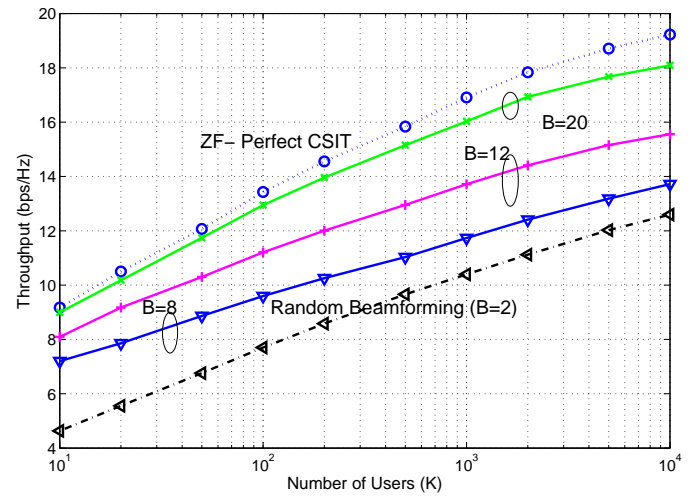

Fig. 2. $M=4, P=10$, System with User Selection

user interference that cannot be overcome by stronger channel magnitudes and ultimately leads to a bounded throughput.

Clearly, the transmitter must have some information about the quantization error in addition to the channel magnitude. In fact, it is sufficient for the CQI to be the following metric, which reflects the channel norm and the quantization error:

$$
\gamma_{i}=\frac{\frac{P}{M}\left\|\mathbf{h}_{i}\right\|^{2} \cos ^{2} \theta_{i}}{1+\frac{P}{M}\left\|\mathbf{h}_{i}\right\|^{2} \sin ^{2} \theta_{i}},
$$

where $\theta_{i}$ is the angle between $\mathbf{h}_{i}$ and its quantization [6]. The quantity $\gamma_{i}$ is the actual received SINR at the $i$-th mobile if the transmitter is able to select a set of $M$ orthogonal quantizations, including $\hat{\mathbf{h}}_{i}$; in other cases, it serves as a very reasonable approximation for the SINR at mobile $i$. If the transmitter performs user selection treating $\sqrt{\gamma_{i}} \hat{\mathbf{h}}_{i}$ as the channel vector of the $i$-th mobile, the optimal $M \log \log K$ throughput growth is achieved. In fact, the feedback-based system is essentially equal to a perfect CSIT system in which user channel norms are distributed according to the distribution of $\gamma_{i}$, (rather than $\frac{P}{M}\left\|\mathbf{h}_{i}\right\|^{2}$ ).

Although optimal throughput scaling is achieved for any $B \geq \log _{2} M$, the actual throughput does depend rather critically on the actual value of $B$, even for large values of $K$. In Fig. 2 throughput is plotted against $K$ for a four antenna system at $10 \mathrm{~dB}(M=4, P=10)$ for various values of $B$. All of the curves, including the bottom curve (labeled random beamforming, which can be shown to be exactly equivalent to the scheme described above when $B=\log _{2} M$ ) have the correct scaling with $K$, but throughput varies significantly as a function of $B$ for every very large systems. As a result, high feedback rates are also required in large downlink channels if performance approaching perfect CSIT is desired.

\section{Practical Quantization Schemes}

While the results of the previous sections were derived assuming random quantization codebooks, which have no particular structure and thus have complexity that grows as $2^{B}$, low complexity quantization schemes for a range of values of 


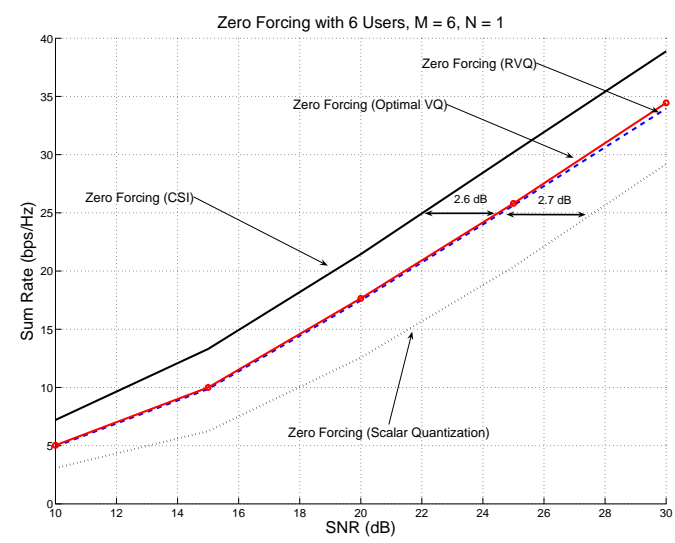

Fig. 3. Scalar Quantization-Based Feedback, $M=K=6$

$B$ are needed for practical implementation. In [10] the low complexity scalar-quantization strategy proposed by Narula et. al [2] is investigated: The $M$-dimensional channel vector $\mathbf{h}_{i}$ is divided by its first element, to yield $M-1$ complex elements, and uniform quantization is performed separately (with uniform bit allocation) on the phase and the inverse tangent of the magnitude of each of these $M-1$ elements.

Although this scheme is extremely simple, it performs reasonably well compared to RVQ. When $M=2$, it can be shown that this scheme provides a bounded rate gap (albeit a large rate gap than RVQ) if it is scaled according to Theorem 2. Although this proof does not yet extend to $M>2$, numerical results indicate that this generalizes to larger values of $M$. In Fig. 3, rates for scalar quantization and RVQ are plotted for a $M=K=6$ system, where bits are scaled according to $B=\frac{M-1}{3} \cdot P_{d B}$. Scalar quantization tracks the RVQ curve, but incurs a power penalty of about $2.7 \mathrm{~dB}$. Also plotted on the figure is an upper bound to the throughput of a system using the same feedback-based ZFBF strategy in conjunction with any vector quantization codebook, assuming that each mobile performs a random rotation of its codebook to ensure isotropic and independent quantizations across mobiles. More sophisticated vector quantization schemes should be able to fill in the gap between this upper bound and the scalar quantization curve.

\section{Multiple Antenna Mobiles}

In this section we describe and compare different methods that utilize a small number of receive antennas at each mobile device (denoted by $N$, with $1<N<M$ ).

\section{A. Block Diagonalization}

When block diagonalization is used on the basis of perfect CSIT, multiple data streams are sent to each mobile and multiuser interference is completely eliminated [8], [9]. Assuming the aggregate number of receive antennas is equal to the number of transmit antennas $(N K=M)$, the transmit signal is given by: $\mathbf{x}=\sum_{i=1}^{K} \mathbf{V}_{i} \mathbf{s}_{i}$, where $\mathbf{V}_{i}$ is the $M \times N$ (unitary) precoding matrix and $\mathbf{s}_{i}$ is the $\mathrm{N}$-dim vector of data symbols intended for the $i$-th mobile. In order to eliminate multi-user interference, the precoding matrices are chosen to satisfy $\mathbf{H}_{j}^{H} \mathbf{V}_{i}=\mathbf{0}$ for all $i \neq j$. If each of the $K N$ receive antennas was treated as a separate user and ZFBF was used, then a separate data stream would be received on each receive antenna, with no correlation between the different signals received at different mobiles or between the $N$ signals received at each mobile. This translates to even stricter restrictions on the precoding matrices, and thus to a loss in throughput [16].

In [10], block diagonalization on the basis of digital channel feedback is analyzed for systems with $K N=M$. In order to appropriately choose precoding matrices, the transmitter only requires knowledge of the subspace spanned by each channel matrix $\mathbf{H}_{i}$. Therefore, each mobile quantizes its channel subspace rather than separately quantizing the $N$ rows of its channel matrix. A quantization codebook consists of $2^{B}$ (unitary) matrices $\left(\mathbf{W}_{1}, \ldots, \mathbf{W}_{2^{B}}\right)$, and the quantizer is the matrix that minimizes the chordal distance $d\left(\mathbf{H}_{i}, \mathbf{W}\right)=\sqrt{\sum_{i=1}^{N} \sin ^{2} \theta_{i}}$, where the $\theta_{i}$ 's are the principal angles between the two subspaces. The idea of random quantization is extended by selecting each quantization matrix in an iid fashion from the set of all $N \times M$ unitary matrices.

Using some of the techniques described in Section III-A as well as subspace quantization bounds from [17], it can be shown that a bounded rate gap between perfect CSIT BD and feedback-based BD can be maintained if the number of feedback bits per mobile is scaled according to [10]:

$$
B \approx N\left(\frac{M-N}{3}\right) P_{d B}+O(1) .
$$

The scaling constant of $N(M-N)$ is due to the fact that the set of $N \times M$ unitary matrices has dimensionality $N(M-N)$.

\section{B. Antenna Combining for Reduced Quantization Error}

An alternative to $\mathrm{BD}$, which results in $N$ streams being sent to each mobile, is to perform antenna combining in order to reduce quantization error and transmit one stream to each mobile [11]. Each mobile linearly combines its $N$ antenna outputs to create a (effective) single antenna output, and quantizes the corresponding effective channel vector. As a result, the system is transformed into a single mobile antenna channel; clearly this is only reasonable when there are $M$ or more mobiles. The advantage of this technique comes from intelligent selection of the linear combiner coefficients, which are chosen to yield an effective vector channel that can be quantized with minimal error.

To understand this method, let us first describe a simpler, albeit considerably less powerful, antenna selection technique. Each mobile has a vector quantization codebook, as described in Section II-A, and separately quantizes the vectors that describe the channel to each of its $N$ antennas (i.e., the $N$ rows of its channel matrix $\mathbf{H}_{i}^{H}$ ). The mobile then selects the antenna with minimum quantization error (angle), and only feeds back the quantization index of the selected antenna 
(using $B$ bits). Only the selected antenna is used for reception, and standard ZFBF is performed since from the transmitter's point of view each mobile has only one antenna. If RVQ is used, this selection method is equivalent to quantizing a single vector channel with $B+\log _{2} N$ bits, thereby effectively increasing the feedback by $\log _{2} N$ bits.

Much more significant gains can be achieved by allowing the antenna to linearly combine its $N$ antenna outputs using any set of combining coefficients; antenna selection can be thought of as restricting the combining weights to be of the form $\left[\begin{array}{lll}1 & 0 & \cdots\end{array}\right]$, etc. When combining is optimally performed, as described in [11], the selected quantizer turns out to be the quantization vector that minimizes the angle between itself and the $N$-dim subspace spanned by $\mathbf{H}_{i}$. Note that the weights are chosen only on the basis of the vector directions because quantization error rather than channel magnitude is more critical for the MIMO downlink. As a result, the technique is very different from standard combining techniques such as those used in limited feedback, point-to-point MIMO systems in which beamforming is performed [3].

Using some of the techniques described in Section III-A as well as properties of random subspaces, it can be shown that a bounded rate gap between perfect CSIT ZF (single antenna) and the throughput achieved with antenna combining can be maintained if the number of feedback bits per mobile is scaled according to [11]:

$$
B \approx \frac{M-N}{3} P_{d B}+O(1) .
$$

\section{Comparison of Techniques}

In this section we compare $\mathrm{BD}$, antenna combining, and simple ZFBF in the small-system regime, i.e., assuming no user selection is performed $(N>1$ with $K N=M)$. The simplest strategy is to treat each receive antenna as a separate user, for the purposes of channel feedback as well as transmission and reception, and send a data stream to each receive antenna (i.e., $N$ streams per mobile) using ZFBF as described in Section III-A. To incur a $3 \mathrm{~dB}$ SNR loss relative to perfect CSIT-ZFBF, $\frac{M-1}{3} P_{d B}$ bits are required per receive antenna, which corresponds to $M\left(\frac{M-1}{3}\right) P_{d B}$ bits in total. If $\mathrm{BD}$ is used, the per mobile feedback rate is given in (9) and the total number of feedback bits is (ignoring constants) $M\left(\frac{M-N}{3}\right) P_{d B}$. The difference between the $M-1$ and $M-N$ terms is precisely the advantage that BD provides over ZFBF; this is due to the fact that the space of $N$-dimensional subspaces is $N(M-N)$, while $N$ one-dimensional subspaces have dimension $N(M-1)$. Antenna combining has the same scaling law as BD (i.e., $M\left(\frac{M-N}{3}\right) P_{d B}$ total bits). However, if antenna combining is used each mobile only receives one data stream and therefore there must be $M$ mobiles with $N$ antennas each (i.e. $K=M$ rather than $K=\frac{M}{N}$ ).

In Table I, feedback requirements for the three strategies are shown for a 6 transmit antenna, 2 receive antenna system. BD and ZFBF apply to systems with 3 mobiles, while antenna combining requires 6 mobiles. The numbers are not the sufficient feedback rates, but instead are numerically computed

\begin{tabular}{l|c|c|c} 
SNR & Block Diag. & Antenna Combining & ZFBF \\
\hline $5 \mathrm{~dB}$ & 4 & 8 & 9 \\
\hline $10 \mathrm{~dB}$ & 10 & 15 & 17 \\
\hline $15 \mathrm{~dB}$ & 17 & 21 & 25 \\
\hline $20 \mathrm{~dB}$ & 24 & 28 & 34 \\
\hline $25 \mathrm{~dB}$ & 30 & 35 & 42 \\
\hline $30 \mathrm{~dB}$ & 37 & 41 & 50 \\
\hline
\end{tabular}

TABLE I

FeEdback Requirements for DifFerent Multiple Mobile ANTENNA STRATEGIES

necessary feedback rates, all relative to a common benchmark of $3 \mathrm{~dB}$ from a perfect CSIT-ZFBF system. BD and antenna combining are seen to significantly outperform ZFBF due to the scaling advantage. Furthermore, BD is seen to require approximately 4 or 5 less bits than antenna combining at each SNR.

\section{REFERENCES}

[1] G. Caire and S. Shamai, "On the achievable throughput of a multiantenna Gaussian broadcast channel," IEEE Trans. Inform. Theory, vol. 49, no. 7, pp. 1691-1706, July 2003.

[2] A. Narula, M. J. Lopez, M. D. Trott, and G. W. Wornell, "Efficient use of side information in multiple antenna data transmission over fading channels," IEEE J. Select. Areas Commun., vol. 16, no. 8, Oct. 1998.

[3] D. Love, R. Heath, and T. Strohmer, "Grassmannian beamforming for multiple-input multiple-output wireless systems," IEEE Trans. Inform. Theory, vol. 49, no. 10, pp. 2735-2747, Oct. 2003.

[4] K. Mukkavilli, A. Sabharwal, E. Erkip, and B. Aazhang, "On beamforming with finite rate feedback in multiple-antenna systems," IEEE Trans. Inform. Theory, vol. 49, no. 10, pp. 2562-2579, Oct. 2003.

[5] N. Jindal, "MIMO broadcast channels with finite-rate feedback," IEEE Trans. on Inform. Theory, vol. 52, no. 11, pp. 5045-5059, Nov. 2006.

[6] T. Yoo, N. Jindal, and A. Goldsmith, "Finite rate feedback MIMO broadcast channels with a large number of users."

[7] M. Sharif and B. Hassibi, "On the capacity of MIMO broadcast channels with partial side information," IEEE Trans. Inform. Theory, vol. 51, no. 2, pp. 506-522, Feb. 2005.

[8] Q. H. Spencer, A. L. Swindlehurst, and M. Haardt, "Zero-forcing methods for downlink spatial multiplexing in multiuser MIMO channels,' Signal Proc., IEEE Trans. on, vol. 52, no. 2, pp. 461-471, 2004.

[9] L. U. Choi and R. D. Murch, "A transmit preprocessing technique for multiuser MIMO systems using a decomposition approach," Wireless Comm., IEEE Trans. on, vol. 3, no. 1, pp. 20-24, 2004.

[10] N. Ravindran and N. Jindal, "MIMO broadcast channels with block diagonalization and finite rate feedback," submitted: ICASSP, Sept. 2006. Available on Arxiv.

[11] N. Jindal, "A feedback reduction technique for MIMO broadcast channels," in Proceedings of Int. Symp. Inform. Theory, July 2006.

[12] W. Santipach and M. Honig, "Asymptotic capacity of beamforming with limited feedback," in Proceedings of Int. Symp. Inform. Theory, July 2004, p. 290.

[13] G. Dimic and N. Sidiropoulos, "On downlink beamforming with greedy user selection: performance analysis and a simple new algorithm," IEEE Trans. Sign. Proc., vol. 53, no. 11, pp. 3857-3868, Oct 2005.

[14] M. Kobayashi and G. Caire, "Joint beamforming and scheduling for a mimo downlink with random arrivals," in Proceedings of Int. Symp. Inform. Theory, July 2006.

[15] T. Yoo and A. Goldsmith, "On the optimality of multi-antenna broadcast scheduling using zero-forcing beamforming," IEEE J. Select Areas Commun., vol. 24, pp. 528-541, March 2006.

[16] J. Lee and N. Jindal, "Dirty paper coding vs. linear precoding for MIMO broadcast channels," in Proceedings of Asilomar Conf. on Signals, Systems, \& Comp., 2006.

[17] W. Dai, Y. Liu, and B. Rider, "Quantization bounds on Grassmann manifolds of arbitrary dimensions and MIMO communications with feedback," IEEE GLOBECOM, 2005. 